\title{
Speed Control of Ultrasonic Motor using a Variable Gain Type PID Control Based on Neural Networks
}

\author{
Shenglin $\mathrm{Mu}^{\mathrm{a}, *}$, Satoru Shibata ${ }^{\mathrm{a}}$, Tomonori Yamamoto ${ }^{\mathrm{a}}$, Shota Nakashima ${ }^{\mathrm{b}}$, Kanya Tanaka $^{\mathrm{c}}$ \\ ${ }^{a}$ Graduate School of Science and Engineering, Ehime University, 3 Bunkyou-chou, Matsuyama, Ehime, Japan \\ ${ }^{\mathrm{b}}$ Graduate School of Sciences and Technology for Innovation, Yamaguchi University, Ube, Yamaguchi, Japan \\ ${ }^{c}$ Department of Electronics and Bioinformatics, School of Science and Technology, Meiji University, Kawasaki, \\ Kanagawa, Japan
}

*Corresponding Author: mu.shenglin.du@ehime-u.ac.jp

\begin{abstract}
In this paper, we introduced an experimental study about a variable gain type PID Control based on neural networks for the speed control of ultrasonic motor. In the proposed method, the neural network controller is employed for tuning the gains in PID controller for speed control in real-time environment. The weights of $\mathrm{NN}$ are designed to be updated by a particle swarm optimization algorithm. This method makes it possible to compensate for the characteristic changes and nonlinearity of ultrasonic motor. By using the particle swarm optimization algorithm in the learning of the neural network unit, the proposed method requires no derivative information of the ultrasonic motor's input and output beforehand; hence its application overcomes the problem of Jacobian estimation in the conventional back propagation method of neural networks. The effectiveness of the proposed method is confirmed by experiments of speed control on the servo system of ultrasonic motor.
\end{abstract}

Keywords: speed control, ultrasonic motor, PID Control, neural networks, particle swarm optimization.

\section{Introduction}

Ultrasonic motor (USM) is a unique kind of motors which employs frictional force generated by high-frequency mechanical vibrations in piezoelectric elements as its drive source. Owing to the driving principle of USM, there are many excellent features such as compactness, low mass, noiselessness, high torque at low speed range, high retention torque, and no electromagnetic noise. Therefore, USMs are expected to be applied as actuators in industrial fields, especially, in the medical and welfare field ${ }^{(1)-(3)}$. However, there are some difficulties in the control of USM. Firstly, due to the friction driven principle, it is hard to get mathematic model of USM. Although some models have been proposed, there is still no accurate model found for high performance control of USM. Secondly, there are characteristic changes and nonlinearity owing to the friction and the characteristics of piezoelectric materials. To solve the problems in USM control, some intelligent schemes based on neural network (NN) have been proposed in recent years. T. Senjyu et al. proposed a direct NN controller ${ }^{(4)}$. In the scheme, a threelayer NN controller using back-propagation (BP) method has been proposed. According to experimental study, the effectiveness of the method was confirmed. In the work of K. Tanaka ${ }^{(5)}$, the researchers proposed an internal model control (IMC)-PID scheme using NN for USM. The method uses the parameter in IMC model as the Jacobian in BP method. However, the Jacobian estimation is a problem we cannot ignore beforehand in practice. Therefore, particle swarm optimization (PSO) has been proposed in resent researches of USM control.

In this paper, an experimental study of speed control based on a variable gain PID using NN combined with PSO is given. $\mathrm{NN}$ in the proposed method is introduced as an autotuning unit for PID controller. Comparing with conventional $\mathrm{NN}$ with back propagation (BP) method for weight updating, PSO is implemented in the proposed method for constructing an intelligent type PID control method in which the gains can be automatically updated to minimize the error in speed 
control to obtain better control than conventional fixed gain type PID control. The effectiveness of the method is confirmed by speed control experiments on an USM servo system.

The paper is organized as follows. The USM and the servo system is introduced in Sect. 2. In Sect.3, the variable gain type PID control using PSO type NN is introduced. Section 4 introduces the experimental setup for speed control of USM. The experimental results and discussions are given in the section. The conclusions are introduced in Sect. 5.

\section{Structure of USM Servo System}

The structure of USM servo system is shown in Fig. 1. The encoder, the magnetic brake which offers the load, and the TWUSM to study, are connected on a common axis in the system as shown in Fig. 2. The position signal fetched by the encoder is transferred to the counter board connected to

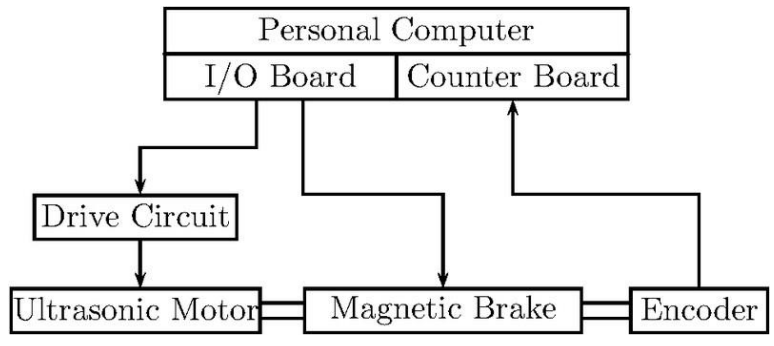

Fig. 1. Structure of USM servo system

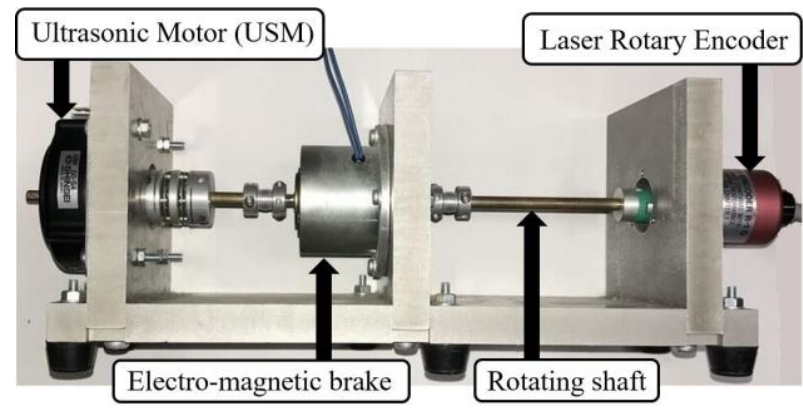

Fig. 2. Photograph of USM, brake and encoder

Table 1 Specifications of USM, encoder and load.

\begin{tabular}{|c|l|}
\hline \multirow{2}{*}{ USM } & Rotational speed: $100[\mathrm{rpm}]$ \\
\cline { 2 - 2 } & Rated Torque: $0.5[\mathrm{~N} \cdot \mathrm{m}]$ \\
\cline { 2 - 2 } & Holding Torque: $1.0[\mathrm{~N} \cdot \mathrm{m}]$ \\
\hline Encoder & Resolution: $0.0011[\mathrm{deg}]$ \\
\hline Magnetic brake & $0 \sim 0.2[\mathrm{~N} \cdot \mathrm{m}]$ \\
\hline
\end{tabular}

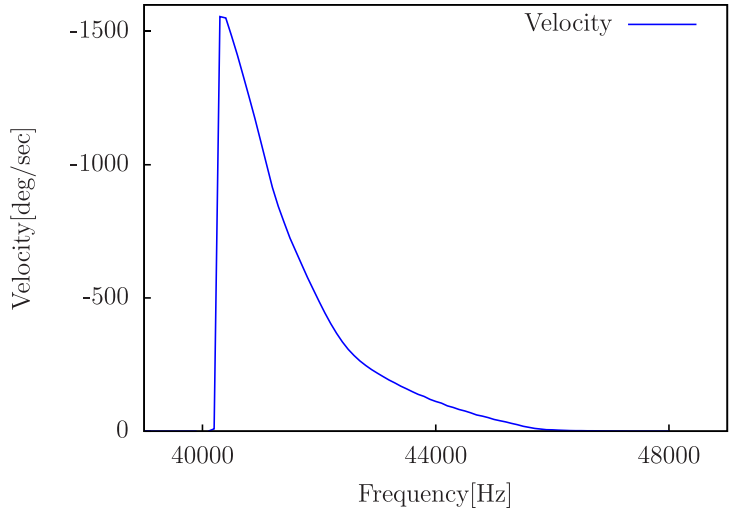

Fig. 3. Rotational velocity characteristics to the frequency control of USM servo sytem.

the PC. The information of the control input calculated from the output and the objective input in the PC is transferred to the driving circuit via the I/O board. The 2-phase control signal will be generated by the driving circuit. In this research, the phase of driving signals in the USM is fixed to 90 [deg], frequency control is applied in the system. There are more specifications of the system shown in Table 1 . The sampling time of the servo system is 1 [ms]. In this study, the range of frequency control is set within the range of $42 \sim 46.5$ $[\mathrm{kHz}]$.

\section{Variable Gain type PID using PSO Type NN}

\subsection{PID control of USM}

Figure 4 shows the block diagram of PID for USM control. In the scheme, $r(k), u(k)$ and $y(k)$ are the object input, the input and the output, respectively. The error between the object input and the output can be estimated as

$$
e(k)=r(k)-y(k)
$$

The PID controller $G_{P I D}\left(z^{-1}\right)$ can be denoted as

$$
G_{P I D}\left(z^{-1}\right)=\frac{K_{P}\left(1-z^{-1}\right)+K_{I}+K_{D}\left(1-z^{-1}\right)^{2}}{1-z^{-1}}
$$

where $K_{P}, K_{I}$ and $K_{D}$ are the gains of the PID controller. By tuning the PID gains, the control performance can be adjusted.

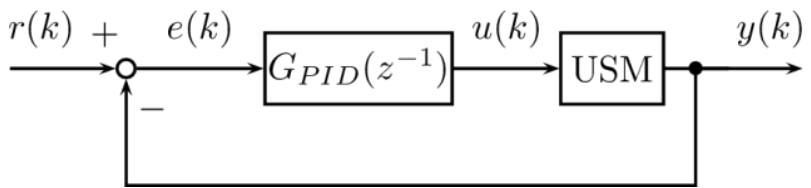

Fig. 4. Block diagram of PID control for USM. 


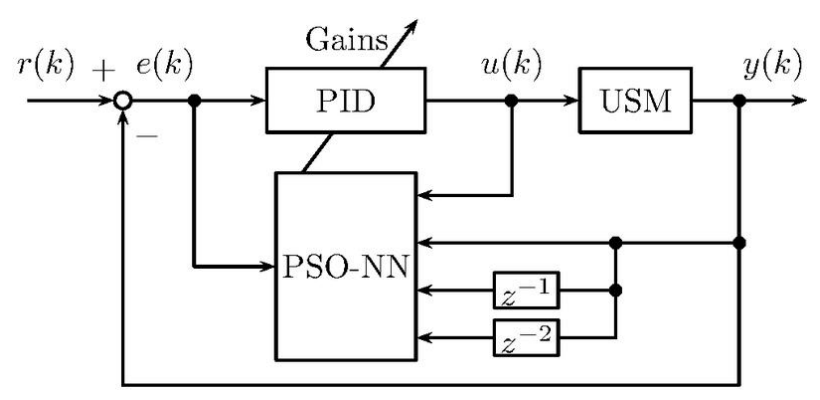

Fig. 5. Proposed scheme of PSO-NN method based on PID control for USM.

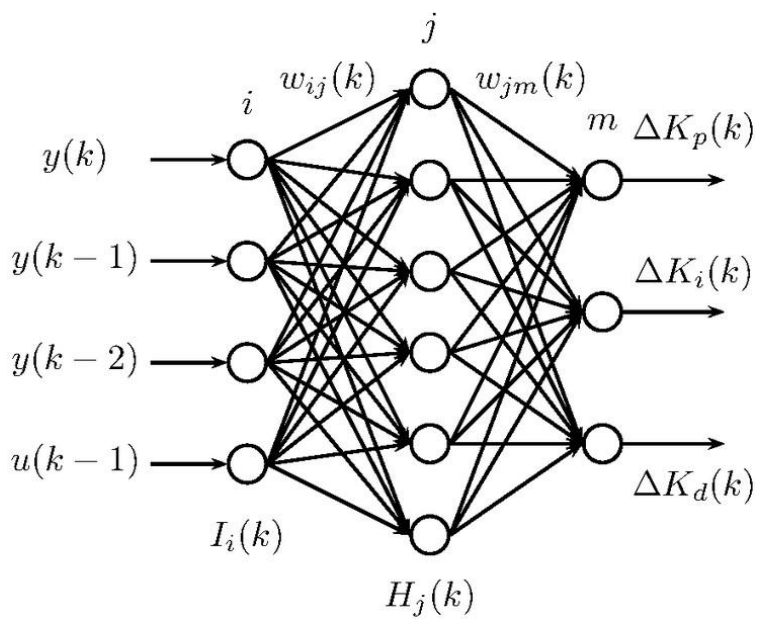

Fig. 6. Topologic structure of NN in the proposed PSO-NN scheme.

The control input $u(k)$ which will be generated by the PID controller can be synthesized as

$$
\begin{aligned}
& u(k)=u(k-1)+\left(K_{P}+K_{I}+K_{D}\right) e(k) \\
& -\left(K_{P}+2 K_{D}\right) e(k-1)+K_{D} e(k-2)
\end{aligned}
$$

\subsection{Variable gain type PID using NN}

In this study, $\mathrm{NN}$ is introduced as the direct intelligent unit to tune the PID controller for the speed control of ultrasonic motor. The proposed NN is a fixed structure in the scheme to tune 3 gains in the PID controller. The learning in NN is designed to be implemented by PSO algorithm. The structure of the proposed scheme is shown in Fig. 5. The topologic structure of NN is shown in Fig. 6. The discrete-time signal of $I_{i}(k)=[y(k), y(k-1), y(k-2), u(k-1)]$ is applied as input in the NN scheme. The variation of PID gains are the output of the scheme. As the activation function, the hyperbolic tangent function shown in Eq. (4) was introduced.

$$
f(x)=\tanh (x)=\frac{e^{x}-e^{-x}}{e^{x}+e^{-x}}
$$

In the proposed method, PSO is introduced to the learning in NN to obtain proper weights in the real-time process. PSO is a global optimization algorithm discovered through the simulation of a simplified social model ${ }^{(6)-(9)}$. As introduced in the previous study, $W_{i}(k)$ means the position of the $i_{\text {th }}$ particle in the $k$ step, $W_{i}(k-1)$ and $W_{i}(k+1)$ mean the position of the $(k-1)$ step and the $(k+1)$ step, respectively. gbest is the best position of the whole swarm, and pbest is the best position of the particle. $V_{i}(k)$, which expresses the position variance, is the velocity of the particle at the $k$ step. It is related to the best positions as the dash lines show in the figure. In NN learning, we define the value of weights as the position information contained by $N$ particles. The weights of $\mathrm{NN}$ are designed to be updated by an inertia weight approach (IWA) type PSO algorithm expressed in Eq. (4) and Eq.(5).

$$
\begin{aligned}
& V_{n+1}=\tau \cdot V_{n}+c_{1} \cdot r_{1} \cdot\left(\text { pbest }_{n}-X_{n}\right) \\
& +c_{2} \cdot r_{2} \cdot\left(\text { gbest }_{n}-X_{n}\right) \\
& X_{n+1}=X_{n}+V_{n}
\end{aligned}
$$

In Eq.(4), $c_{1}$ and $c_{2}$ are two positive constants, $r_{1}$ and $r_{2}$ are two random numbers within [0,1]. In Eq.(5), the weight factor which balances the global and local search, is calculated as follows.

$$
\tau=\tau_{\max }-\frac{\tau_{\max }-\tau_{\min }}{\text { episode }_{\max }} \cdot \text { episode }
$$

where episode means the number of current iterations, episode $_{\max }$ presents the max iteration number in the searching. $\tau_{\max }$ and $\tau_{\min }$ are the weight factor's maximum and minimum value, respectively. By using the IWA type PSO in the learning of $\mathrm{NN}$, the weights can be updated to minimize the error in the control process. To evaluate the performance of the possible solutions contained by the particles, the fitness of the solutions was designed as

$$
\text { fitness }=\frac{1}{1+e(k)^{2}}
$$

\section{Experimental Study}

In the experiments, a group of ramp signals were employed as the objective input to evaluate the control performance of speed control on the servo system. The objective speed was set to $15[\mathrm{deg} / \mathrm{s}]$ and $35[\mathrm{deg} / \mathrm{s}]$ for comparison. Figure 7(a) shows the response of the fixed gain PID control method with the gains of $K_{P}=5.0, K_{I}=2.0$, 


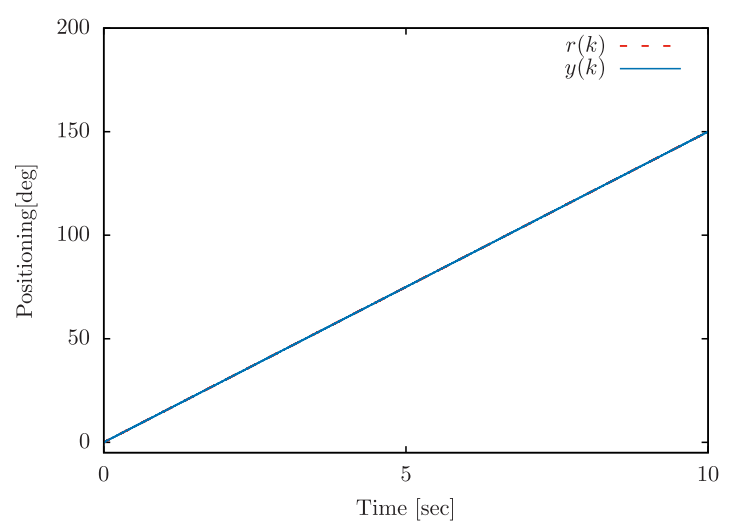

(a) Response of constant speed control of 15 [deg/s]

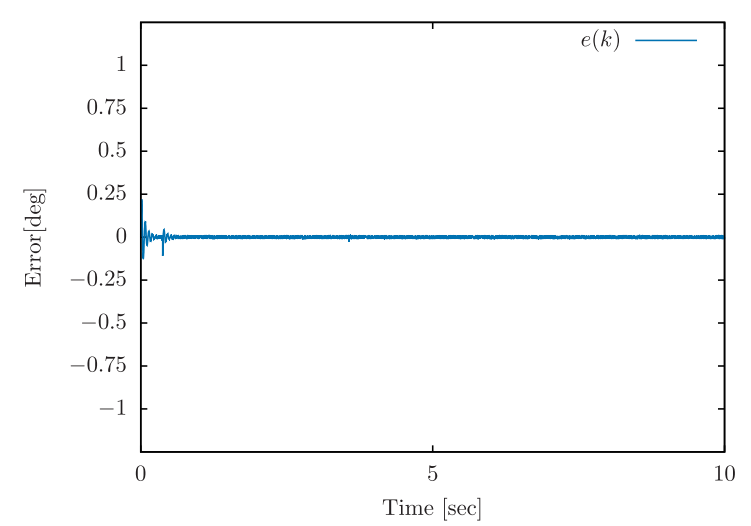

(b) Error variation in the speed control

Fig. 7. Experimental results of fixed gain type PID control with the objective speed of 15 [deg/s]

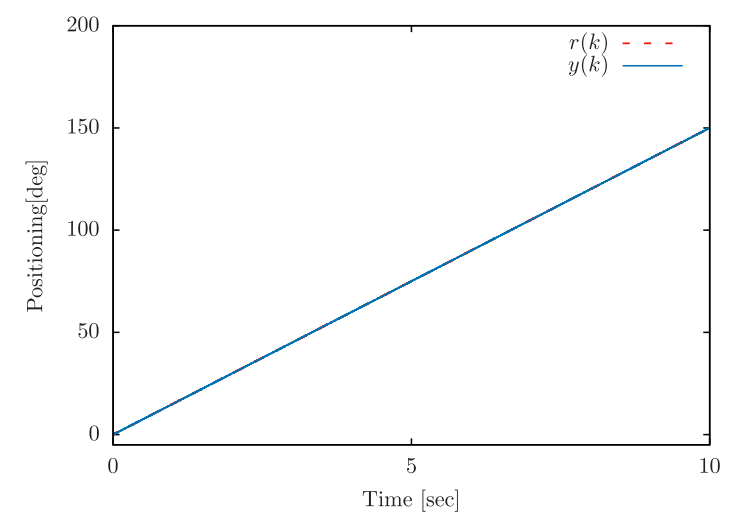

(a) Response of constant speed control of 15 [deg/s]

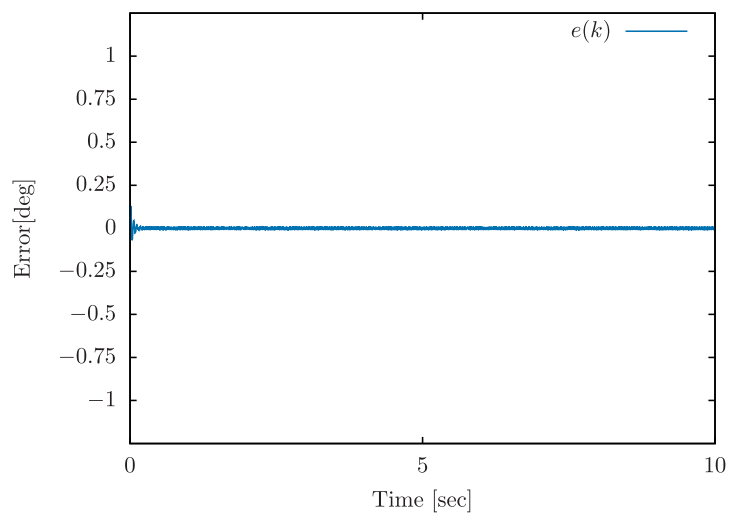

(b) Error variation in the speed control

Fig. 8. Experimental results of the variable gain type PID control with the objective speed of 15 [deg/s]

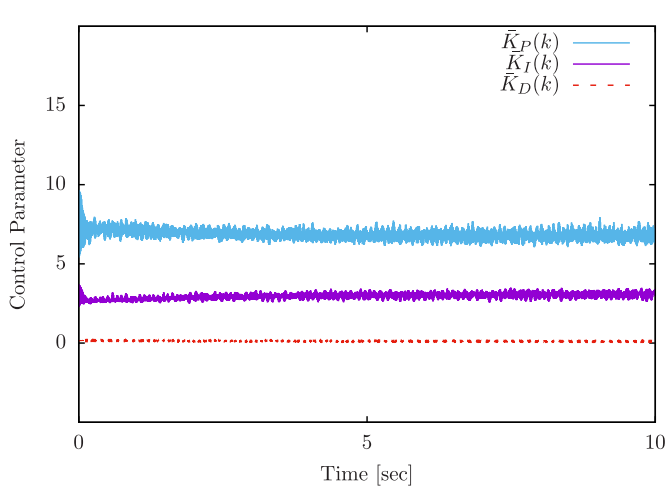

(a) Gain variation in the PID control

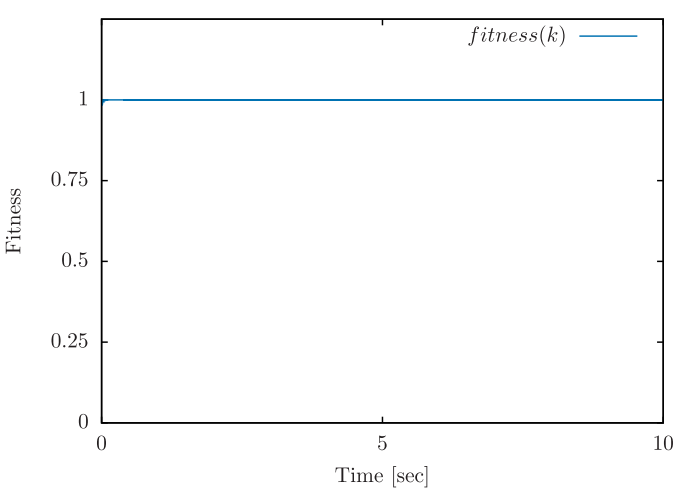

(b) Fitness variation in the PSO.

Fig. 9. Parameter variation in the variable gain type PID control with the objective speed of 15 [deg/s]

$K_{D}=0.1$. The output signal $y(t)$ follows the object input $r(t)$ in the $10 \mathrm{sec}$ control process. The variation of error signal is shown in Fig. 7(b). It shows that there was some error in the control process. Figure 8(a) shows the response of the proposed PSO-NN type variable gain PID control method. In the response signal, the same level response results were shown. Comparing with the error signal shown in Fig. 6(b), the error signal of the proposed method shown in Fig. 7(b) gave better performance. The parameter variation in the proposed method is shown in Fig. 9. Figure 8(a) shows the 


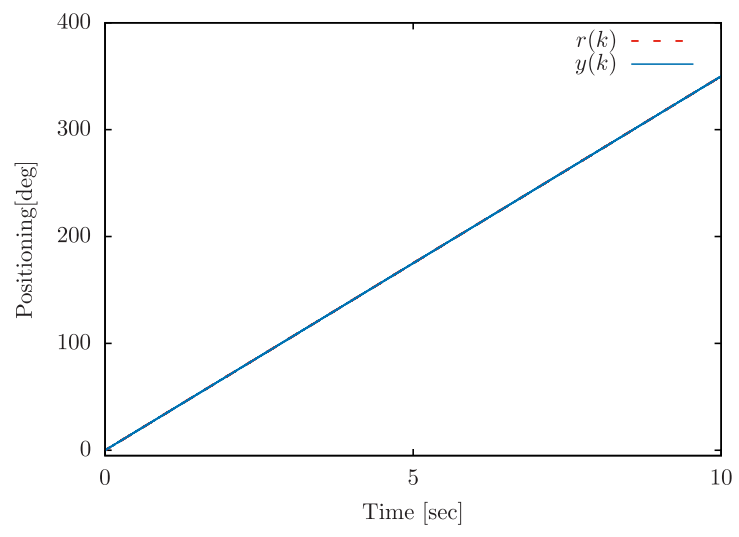

(a) Response of constant speed control of $35[\mathrm{deg} / \mathrm{s}]$

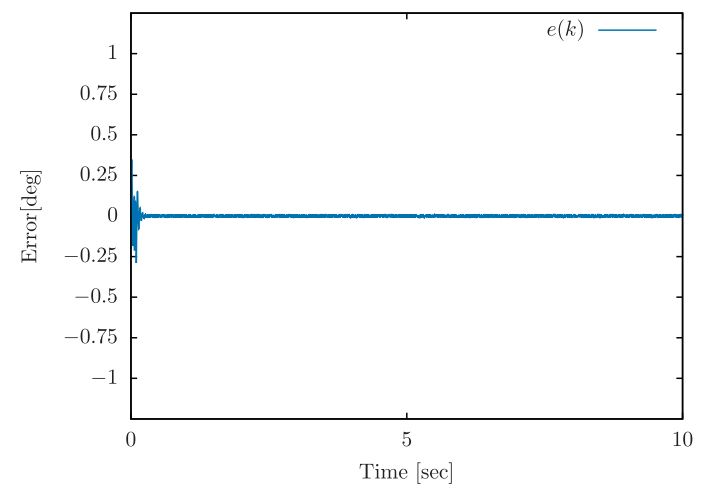

(b) Error variation in the speed control

Fig. 10. Experimental results of fixed gain type PID control with the objective speed of 35 [deg/s]

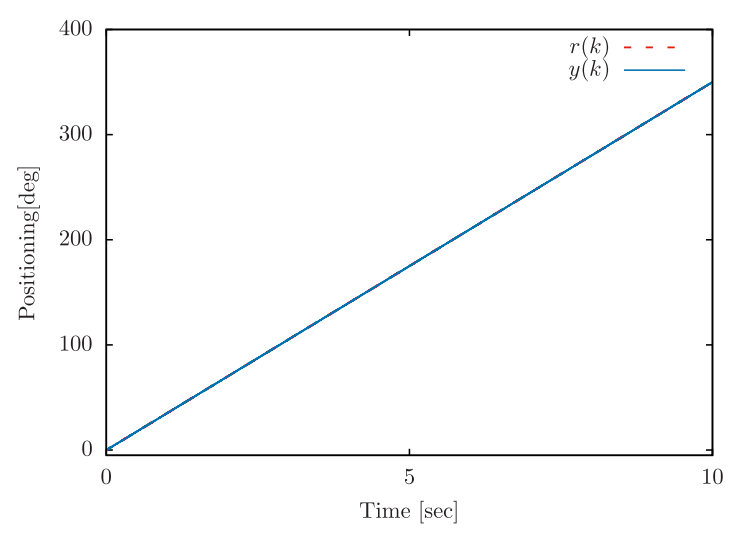

(a) Response of constant speed control of $35[\mathrm{deg} / \mathrm{s}]$

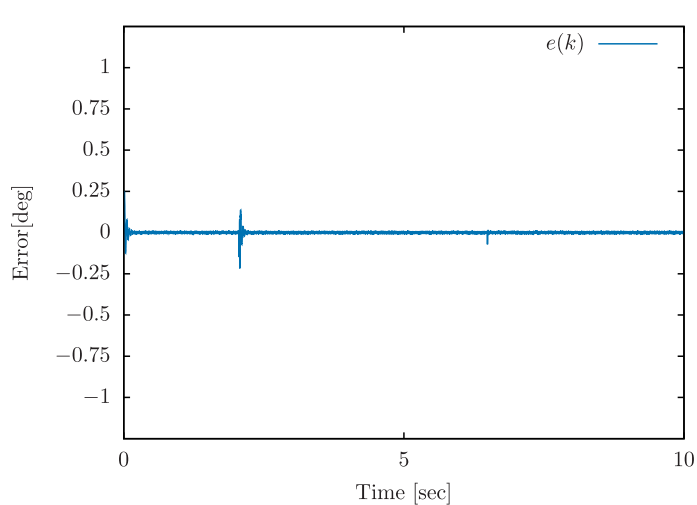

(b) Error signal of varied triangle signal response

Fig. 11. Experimental results of the variable gain type PID control with the objective speed of 35 [deg/s]

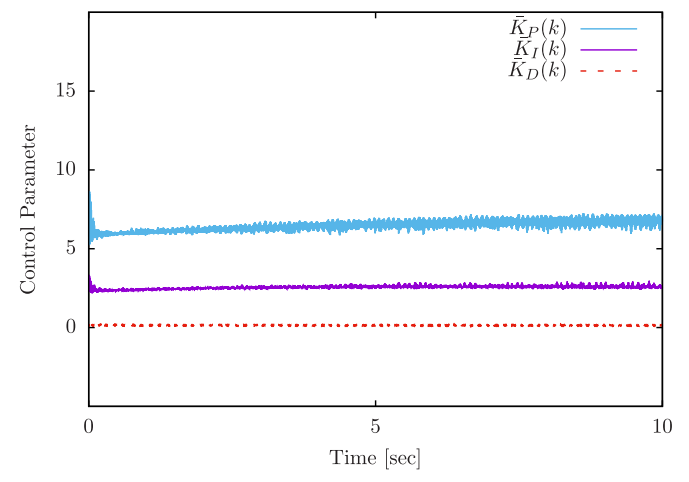

(a) Gain variation in the PID control

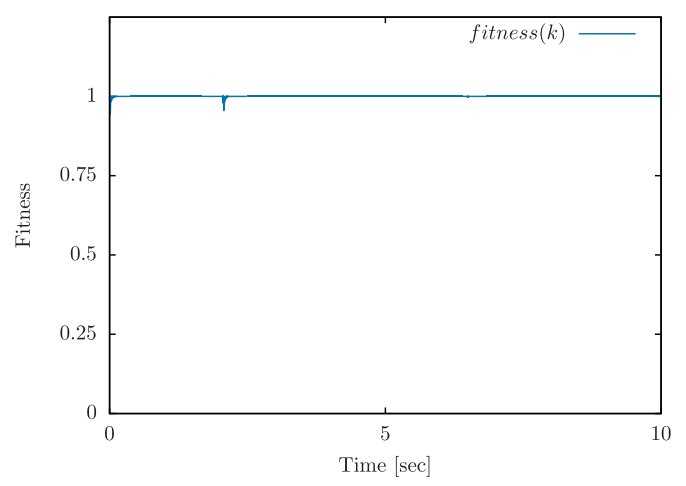

(b) Fitness variation in the PSO.

Fig. 12. Parameter variation in the variable gain type PID control with the objective speed of 35 [deg/s]

variation of PID gains in the real-time control. The continuous variation of gains offered better control performance than conventional fixed gain PID method. Meanwhile, the fitness in PSO algorithm, which is shown in Fig. 8(b), also leads to the same conclusion as the error signals showed above. In the speed control with the objective input of 35 [deg/s], the control output is shown in Fig. 9. The response and the error variation are shown in the figures of (a) and (b), respectively. The output of the proposed method is shown in Fig. 11 and Fig. 12. Figure 11(a) shows the 
response of the proposed PSO-NN type variable gain PID control method. Figure 11(b) shows the error variation, in which we can see that the proposed method is with smaller error than the fixed gain type PID control. However, there were some noise causing some error in the process. The parameter variation in the proposed method is shown in Fig. 12.

\section{Conclusions}

In this paper, a variable gain type PID control scheme is studied for the speed control of USM. In this method, PSO algorithm is applied in NN's learning, and the output of NN is used for tuning the PID controller. The proposed method overcomes the Jacobian estimate problem of conventional BP type NN. The effectiveness of the proposed method is confirmed by experiments using USM servo system.

\section{References}

(1) T. Kenjo and T. Sashida: "An Introduction of Ultrasonic Motor" Oxford Science Publications, 1993.

(2) K. Adachi: "Actuator with friction drive: Ultrasonic motor", The Japan Society of Mechanical Engineers, Vol. 108: pp. 48-51, 2005

(3) C. Zhao: "Ultrasonic Motor - Technologies and
Applications", Science Press Beijing and SpringerVerlag Berlin Heidelberg, Beijing, 2011

(4) T. Senjyu, H. Miyazato, and K. Uezato. "Position control of ultrasonic motor using neural network." Transaction of the Institute of Electrical Engineers of Japan D, Vol.116:1059-1066, 1996

(5) K. Tanaka, Y. Yoshimura, Y. Wakasa, T. Akashi, M. Oka and $\mathrm{S}$. Mu, Variable gain type intelligent PID control for ultrasonic motor, The Japan Society Applied Electromagnetics and Mechanics, vol.17, pp.107-113, 2009.

(6) J. Kennedy, R. Eberhart: "Swarm Intelligence", Morgan Kaufmann Publishers, 2001

(7) J. Kennedy and R. Eberhart: "Particle Swarm Optimization”, Proc. IEEE Int. Conf. Neural Networks, Perth, Australia, pp. 1942-1948, 1995

(8) M. Clerc and J. Kennedy: "The Particle Swarm: Explosion, Stability, and Convergence in a MultiDimensional Complex Space", IEEE Trans. Evolutionary Computation, Vol. 6, No. 1, pp.58-73, 2002

(9) M. Ito and M. Tanaka: "A Study of Particle Swarm Optimization for Neural Network Training”, A publication of Electronics, Information and System Society, pp. 1087-1089, 2005 